\title{
ASYMPTOTIC ANALYSIS OF THE CLOSED QUEUEING STRUCTURE WITH TIME-DEPENDENT SERVICE PARAMETERS AND SINGLE-TYPE MESSAGES
}

\author{
Mikhail Matalytski ${ }^{1}$, Tatiana Rusilko ${ }^{2}$, Andrey Pankov ${ }^{3}$ \\ ${ }^{1}$ Institute of Mathematics, Czestochowa University of Technology \\ Czestochowa, Poland \\ 2,3 Grodno State University, Grodno, Belarus \\ I'm.matalytski@gmail.com, ${ }^{2}$ romaniuk@grsu.by,3a.pankov@gmail.com
}

\begin{abstract}
A closed queueing structure is considered in the paper; the number of single-type messages is not constant and depends on time. The route of messages is given by an arbitrary stochastic matrix of transition probabilities. An asymptotic analysis of this structure in case of large number of service requests is conducted. The service parameters of each queueing system of this structure, as well as the probability of messages transition between systems, depend on time. A system of ordinary differential equations to calculate the average relative number of messages in each queueing system, depending on the time, was obtained.
\end{abstract}

Keywords: queueing network, differential equation, asymptotic analysis

\section{Introduction}

Queueing networks (QN) are often used as mathematical models of various technical, economical and other processes and objects. Development of new approaches and methods of modern QN theory are often dictated by the need to consider a number of features of objects being modeled. According to the definition of a closed $\mathrm{QN}$, the total number of messages it serves, is constant. In practice, the total number of messages in many model objects changes over time. Due to the above new mathematical object - a closed queueing structure was put into consideration, that is closed queueing network, the total number of services messages is specified by a function of time [1].

Such queueing structures are used as models of the processing of customer claims for an insurance company $[1,2]$. However, the parameters of customer service in the insurance companies are not constant throughout the period of time. In this regard, there is a problem of study of closed queueing structure provided depending on time of the intensities of service requests, the number of service lines and transition probabilities between the systems of the structure. 


\section{Diffusion approximation of closed queueing structure with single type of messages}

Consider a closed queueing structure consisting of $n+1$ queueing systems (QS) $S_{0}, S_{1}, \ldots, S_{n}$ where the total number of single-type messages at time $t$ is described by function of time $K(t), K(t) \leq M$. Suppose that the parameters of services of this structure depend on time $t, t \in[0, T]$. Let the number of service lines in the system $S_{i}$ at time $t$ be described by function of time $m_{i}(t)$, that returns integer values, $i=\overline{0, n}$. Suppose that the number of service lines in the system $S_{0}$ is $M$. The service time by each line of system $S_{i}$ is distributed exponentially with a time-dependent mean value $\mu_{i}^{-1}(t)$, where $\mu_{i}(t) \geq 0, i=\overline{0, n}$. Requests for service are selected accordingly to the discipline FIFO. Message that completed servicing in the system $S_{i}$ goes to the queue of system $S_{j}, i, j=\overline{0, n}$, with the probability $p_{i j}(t)$. The transition matrix $P(t)=\left\|p_{i j}(t)\right\|$ is the matrix of transition probabilities of an irreducible Markov chain, and depends on the time in the general case, $0 \leq p_{i j}(t) \leq 1, \sum_{j=0}^{n} p_{i j}(t)=1$. The main objective of the study of closed queueing structure described here is the asymptotic analysis of the Markov process describing its behavior, with a large number of messages. The state of the network at time $t$ is described by the vector

$$
k(t)=\left(k_{1}(t), k_{2}(t), \ldots, k_{n}(t)\right),
$$

where $k_{i}(t)$ - the number of messages in the system $S_{i}$ at time $t, t \in[0, T], i=\overline{1, n}$, which forms $n$-dimensional Markov process with continuous time and finite number of states. Because the queueing structure is closed, it is obvious that the number of messages in the system $S_{0}$ at time $t$ is equal to $k_{0}(t)=K(t)-\sum_{i=1}^{n} k_{i}(t)$.

Theorem. The probability density function $p(x, t)$ of vector of relative variables $\xi(t)=\left(\frac{k_{1}(t)}{K(t)}, \frac{k_{2}(t)}{K(t)}, \ldots, \frac{k_{n}(t)}{K(t)}\right)$ provided that it is twice differentiable by $t$ and twice piecewise continuously differentiable by $x_{i}, i=\overline{1, n}$, satisfies up to $\mathrm{O}\left(\varepsilon^{2}(t)\right)$, where $\varepsilon(t)=\frac{1}{K(t)}$, equation

$$
\begin{gathered}
\frac{\partial p(x, t)}{\partial t}=-\sum_{i=1}^{n} \frac{\partial}{\partial x_{i}}\left(A_{i}(x, t) p(x, t)\right)+\frac{\varepsilon(t)}{2} \sum_{i, j=1}^{n} \frac{\partial^{2}}{\partial x_{i} \partial x_{j}}\left(B_{i j}(x, t) p(x, t)\right)+ \\
+n \varepsilon(t) K^{\prime}(t) p(x, t)
\end{gathered}
$$


where

$$
\begin{gathered}
A_{i}(x, t)=\sum_{j=0}^{n} \mu_{j}(t) p_{j i}^{*}(t) \min \left(l_{j}(t), x_{j}\right), \\
B_{i i}(x, t)=\sum_{j=0}^{n} \mu_{j}(t) r_{j i}(t) \min \left(l_{j}(t), x_{j}\right), \\
B_{i j}(x, t)=-\mu_{i}(t) p_{i j}(t) \min \left(l_{i}(t), x_{i}\right), \\
p_{j i}^{*}(t)=r_{j i}(t)=p_{j i}(t), \quad i \neq j ; p_{i i}^{*}(t)=-r_{i i}(t)=-1+p_{i i}(t), \quad i=j .
\end{gathered}
$$

Proof. Consider all possible transitions of the process during time $\Delta t$ into the state $k(t+\Delta t)=(k, t+\Delta t)$ :

- from state $\left(k+I_{i}-I_{j}, t\right)$ can get into the state $(k, t+\Delta t)$ with probability

$$
\mu_{i}(t) \min \left(m_{i}(t), k_{i}(t)+1\right) p_{i j}(t) \Delta t+o(\Delta t), i, j=\overline{0, n}
$$

- from state $(k, t)$ - with probability

$$
1-\sum_{i=0}^{n} \mu_{i}(t) \min \left(m_{i}(t), k_{i}(t)\right) \Delta t+o(\Delta t) ;
$$

- from other states - with probability $o(\Delta t)$. Here $I_{i}-n$-dimensional zero-vector with exception that $i$-th component equals 1 , when $1 \leq i \leq n, I_{0}$-n-dimensional zero-vector.

Applying the formula of total probability, we can write the system of difference equations for the state probabilities $P(k, t)$ :

$$
\begin{gathered}
P(k, t)=\sum_{i, j=0}^{n} \mu_{i}(t) p_{i j}(t) \min \left(m_{i}(t), k_{i}(t)+1\right) P\left(k+I_{i}-I_{j}, t\right) \Delta t+ \\
+\left(1-\sum_{i, j=0}^{n} \mu_{i}(t) p_{i j}(t) \min \left(m_{i}(t), k_{i}(t)\right) \Delta t\right) P(k, t)+o(\Delta t) .
\end{gathered}
$$

Using the limit for $\Delta t \rightarrow 0$, we obtain a system of difference-differential Kolmogorov equations for the state probabilities:

$$
\begin{gathered}
\frac{d P(k, t)}{d t}=\sum_{i, j=0}^{n} \mu_{i}(t) p_{i j}(t) \min \left(m_{i}(t), k_{i}(t)\right)\left(P\left(k+I_{i}-I_{j}, t\right)-P(k, t)\right)+ \\
+\sum_{i, j=0}^{n}\left[\mu_{i}(t) p_{i j}(t)\left(\min \left(m_{i}(t), k_{i}(t)+1\right)-\min \left(m_{i}(t), k_{i}(t)\right)\right) P\left(k+I_{i}-I_{j}, t\right) .\right.
\end{gathered}
$$


Next, we consider the case of a large number of messages in the network, $K(t) \gg 1$, and move to the vector of relative variables $\xi(t)=\left(\frac{k(t)}{K(t)}\right)$, it's possible that the values belong to the bounded closed set $G=\left\{x=\left(x_{1}, x_{2}, \ldots, x_{n}\right): x_{i} \geq 0, i=\overline{1, n}, \sum_{i=1}^{n} x_{i} \leq 1\right\}$, where they are located at the nodes of $n$-dimensional lattice at a distance $\varepsilon(t)=\frac{1}{K(t)}$ from each other. With increasing values of function $K(t)$ "filling density" of the set $G$ by possible components of this vector increases, and it becomes possible to believe that vector has continuous distribution with the probability density $p(x, t)=K^{n}(t) P(x K(t), t)=K^{n}(t) P(k, t), x \in G$, where $p(x, t)$ has meaning of the probability density function of the random vector $\xi(t)$.

Denoted $e_{i}=\varepsilon(t) I_{i}, i=\overline{1, n}, c(u)=\left\{\begin{array}{l}1, u>0, \\ 0, u \leq 0\end{array}\right.$. Note that $\min (u, v+1)=\min (u, v)+c(u-v)$, $c(u-v)=\frac{\partial \min (u, v)}{\partial v}$, because $\min (u, v)=\left\{\begin{array}{l}v, u \geq v, \\ u, u<v\end{array}\right.$. Introduce the notation $l_{i}(t)=\frac{m_{i}(t)}{K(t)}$, $i=\overline{1, n}$. Rewriting the system of equations (2) for density $p(x, t)$, we obtain

$$
\begin{aligned}
& \frac{\partial p(x, t)}{\partial t}=\sum_{i, j=0}^{n} K(t) \mu_{i}(t) p_{i j}(t) \min \left(l_{i}(t), x_{i}\right)\left(p\left(x+e_{i}-e_{j}, t\right)-p(x, t)\right)+ \\
& +\sum_{i, j=0}^{n} \mu_{i}(t) p_{i j}(t) \frac{\partial \min \left(l_{i}(t), x_{i}\right)}{\partial x_{i}} p\left(x+e_{i}-e_{j}, t\right)+n \varepsilon(t) K^{\prime}(t) p(x, t) .
\end{aligned}
$$

We represent the right-hand side of this set of equations, up to terms of order of smallness $\varepsilon^{2}(t)$. We will assume that $p(x, t)$ is differentiable both at $t$ and twice continuously differentiable at $x_{i}, i=\overline{1, n}, \frac{\partial p(x, t)}{\partial x_{0}}=0$. Then the following Taylor series expansion takes place:

$$
\begin{gathered}
p\left(x+e_{i}-e_{j}, t\right)=p(x, t)+\varepsilon(t)\left(\frac{\partial p(x, t)}{\partial x_{i}}-\frac{\partial p(x, t)}{\partial x_{j}}\right)+ \\
+\frac{\varepsilon^{2}(t)}{2}\left(\frac{\partial^{2} p(x, t)}{\partial x_{i}^{2}}-2 \frac{\partial^{2} p(x, t)}{\partial x_{i} \partial x_{j}}+\frac{\partial^{2} p(x, t)}{\partial x_{j}^{2}}\right)+o\left(\varepsilon^{2}(t)\right), i, j=\overline{1, n} .
\end{gathered}
$$


Using the last expansion and the fact that $\varepsilon(t) K(t)=1$, we obtain:

$$
\begin{gathered}
\frac{\partial p(x, t)}{\partial t}=n \varepsilon(t) K^{\prime}(t) p(x, t)+ \\
+\sum_{i, j=0}^{n} \mu_{i}(t) p_{i j}(t) \min \left(l_{i}(t), x_{i}\right)\left[\left(\frac{\partial p(x, t)}{\partial x_{i}}-\frac{\partial p(x, t)}{\partial x_{j}}\right)+\right. \\
\left.+\frac{\varepsilon(t)}{2}\left(\frac{\partial^{2} p(x, t)}{\partial x_{i}^{2}}-2 \frac{\partial^{2} p(x, t)}{\partial x_{i} \partial x_{j}}+\frac{\partial^{2} p(x, t)}{\partial x_{j}^{2}}\right)\right]+ \\
+\sum_{i, j=0}^{n} \mu_{i}(t) p_{i j}(t) \frac{\partial \min \left(l_{i}(t), x_{i}\right)}{\partial x_{i}}\left[p(x, t)+\varepsilon(t)\left(\frac{\partial p(x, t)}{\partial x_{i}}-\frac{\partial p(x, t)}{\partial x_{j}}\right)+\right. \\
\left.+\frac{\varepsilon^{2}(t)}{2}\left(\frac{\partial^{2} p(x, t)}{\partial x_{i}^{2}}-2 \frac{\partial^{2} p(x, t)}{\partial x_{i} \partial x_{j}}+\frac{\partial^{2} p(x, t)}{\partial x_{j}^{2}}\right)\right]+o\left(\varepsilon^{2}(t)\right) .
\end{gathered}
$$

Using the notations (3), (4), the last equation can be written as (2) up to terms of order of smallness $\varepsilon^{2}(t)$.

\section{Calculation of the average relative number of messages in systems of closed queueing structure}

Partial differential equation of second order (2) belongs to the parabolic equations. Note that when $K(t)=K$ this equation is the same as Kolmogorov-Fokker-Planck equation for the probability distribution of a Markov process $\xi(t)$.

Applying to (2) the method of the Gaussian approximation [1], we get system of ordinary differential equations for the components of vector of the average relative number of messages in systems of the queueing structure $n(t)=\left(n_{1}(t), n_{2}(t), \ldots, n_{n}(t)\right)$, where $n_{i}(t)=M\left(\xi_{i}(t)\right)=M\left(\frac{k_{i}(t)}{K(t)}\right)$. However, since the right-hand side of (2) contains the piece-wise linear terms of the form $\min \left(l_{i}(t), x_{i}\right)$, we use the phase space and solve the obtained system of difference-differential equations in the regions where its right-hand parts are linear.

Let $\Omega(t)=\{1,2, \ldots, n\}$ - the set of components indices of vector $n(t)$. We divide $\Omega(t)$ into two disjoint sets $\Omega_{0}(t)$ and $\Omega_{1}(t)$ :

$$
\Omega_{0}(t)=\left\{i: l_{i}(t)<n_{i}(t) \leq 1\right\}, \quad \Omega_{1}(t)=\left\{j: 0 \leq n_{j}(t) \leq l_{j}(t)\right\} .
$$


For a fixed $t$ number of partitions of this type is equal to $2^{n}$. Each partition on set $G(t)=\left\{n(t): n_{i}(t) \geq 0, \sum_{i=1}^{n} n_{i}(t) \leq 1\right\}$ will define disjoint sets $G_{\tau}(t)$ such that

$$
\begin{gathered}
G_{\tau}(t)=\left\{n(t): l_{i}(t)<n_{i}(t) \leq 1, i \in \Omega_{0}(t) ; 0 \leq n_{j}(t) \leq l_{j}(t), j \in \Omega_{1}(t) ; \sum_{c=1}^{n} n_{c}(t) \leq 1\right\}, \\
\tau=1,2, \ldots, 2^{n}, \bigcup_{\tau=1}^{2^{n}} G_{\tau}(t)=G(t) .
\end{gathered}
$$

Then the system of equations for the components of vector $n(t)$ for each region $G_{\tau}(t)$ can be written in explicit form. For example, for region

$$
A: \Omega_{1}(t)=\{1,2, \ldots, n-1\}, \Omega_{0}(t)=\{n\},
$$

that corresponds to the absence of queues in average in systems $S_{i}, i=\overline{0, n-1}$, and presence of queue in system $S_{n}$, we obtain the following set of differential equations:

$$
\begin{aligned}
& n_{i}^{\prime}(t)=\mu_{0}(t) p_{0 i}^{*}(t)\left(1-\sum_{i=1}^{n} n_{i}(t)+\varepsilon(t)\right)+ \\
& +\sum_{j=1}^{n-1} \mu_{j}(t) p_{j i}^{*}(t) n_{j}(t)+\mu_{n}(t) p_{n i}^{*}(t) l_{n}(t) .
\end{aligned}
$$

The solution of (7) for certain initial conditions allows one to find the average relative number $n_{i}(t)$, therefore the average number is $K(t) n_{i}(t)$ of messages for each system of queueing structure.

\section{Example}

Consider a closed queueing structure consisting of 4 systems $S_{0}, S_{1}, S_{2}, S_{3}$, the total number of single-type messages circulating there is described by the function $K(t)=15 \sin (6 t)+30000$. We define the following probabilities of transitions between the queueing systems:

$$
\begin{gathered}
p_{03}(t)=1-p_{01}(t), p_{10}(t)=1-p_{12}(t), \\
p_{21}(t)=1-p_{23}(t), p_{32}(t)=1-p_{30}(t), \\
p_{01}(t)=p_{12}(t)=0.4(\cos (t)+1), p_{23}(t)=0.3(\sin (t)+1), \\
p_{30}(t)=0.1(\sin (t)+1), p_{i j}(t)=0 \text { in remaining cases. }
\end{gathered}
$$


We define the initial distribution of messages by systems of the structure as follows: $n_{i}(0)=0, i=1,2, n_{3}(t)=0.1$. Suppose that there are no queues in systems $S_{i}$, $i=1,2$, and there is a queue in system $S_{3}$. In this case the system of differential equations (7) would be:

$$
\left\{\begin{array}{l}
n_{1}^{\prime}(t)=\mu_{0}(t) p_{01}(t)\left(1-\sum_{i=1}^{n} n_{i}(t)+\varepsilon(t)\right)+\mu_{2}(t) p_{21}(t) n_{2}(t)-\mu_{1}(t) n_{1}(t) \\
n_{2}^{\prime}(t)=\mu_{1}(t) p_{12}(t) n_{1}(t)-\mu_{2}(t) n_{2}(t)+\mu_{3}(t) p_{32}(t) l_{3}(t) \\
n_{3}^{\prime}(t)=\mu_{0}(t) p_{03}(t)\left(1-\sum_{i=1}^{n} n_{i}(t)+\varepsilon(t)\right)+\mu_{2}(t) p_{23}(t) n_{2}(t)-\mu_{3}(t) l_{3}(t)
\end{array}\right.
$$

We define the intensity of servicing of messages in queueing systems as the following functions:

$$
\begin{gathered}
\mu_{1}(t)=0.0002+0.0007 t, \quad \mu_{2}(t)=0.00055+0.0004 t \\
\mu_{3}(t)=0.0005+0.00015 t, \quad \mu_{0}(t)=0.00025+0.00015 t
\end{gathered}
$$

Let the number of service lines in the system $S_{3}$ equal

$$
m_{3}(t)=[2 \sin (5 t)+3]
$$

where $[\cdot]$ - integer part of the number.

System (8) is a system of linear differential equations with non-constant coefficients. It is difficult to find an analytical solution of this system. For a numerical solution of differential equations of the form (8) mathematical computing software Maple can be applied.

Figure 1 shows graphically the behavior of $n_{1}(t)$ depending on time. It is possible to explore in the same way the remaining components of vector $n(t)$, which are the solution of (8).

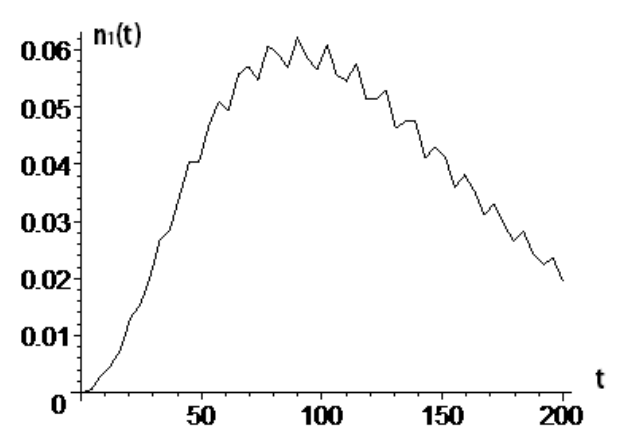

Fig. 1. Graph of $n_{1}(t)$ 


\section{Conclusion}

The considered method of calculation of the average relative number of messages in systems $S_{i}, i=\overline{1, n}$, is valid only for a high load of queueing structure, i.e. in the case when the function $K(t)$ returns large values. Accuracy of the method increases with the total number of servicing messages.

\section{References}

[1] Matalytski M., Rusilko T., Mathematical Analysis of Stochastic Models of Claims Processing in Insurance Companies, GrSU, Grodno 2007.

[2] Rusilko T., Research of stochastic model of insurance company's customer claims with time-dependent servicing parameters, Vestnik GrSU 2011, 3, 167-174.

[3] Medvedev G., On optimization of closed queueing system, Proceedings of the Academy of Sciences of the USSR, Technical Cybernetics 1975, 6, 65-73. 